Methods PBL from active SLE patients and age- and sexmatched healthy controls were assessed for increased expression of the $\mathrm{T}$ cell homeostatic proliferation phenotype determined from our murine studies: CD45RO+PD-1+SLAMF7 +IL-7R-alpha-. The same cells were analyzed for MAVS oligomerization. Young lupus-prone MRL-lpr mice were treated for 11 weeks with the mitochondrially targeted antioxidant MitoQ.

Results Reactive oxygen species (ROS) is sufficient to drive MAVS oligomerization and IFN-I production in cell lines and primary human PBL. However, PBL from SLE patients manifested spontaneous MAVS oligomerization, which paralleled the levels of serum INF-I and SLEDAI score (figure 1). Furthermore, SLE patients had evidence of increased $\mathrm{T}$ cell homeostatic proliferation. In addition, SLE patients had evidence of increased I cell homeostatic proliferation, based on increased IL-7R- Ki67 + T cells. Very similar findings were observed in lupus prone MRL-lpr mice. Treatment of MRL-lpr mice over 11 weeks with the mitochondrially-targeted anti-oxidant MitoQ inhibited MAVS oligomerization, reduced serum IFN-I, improved dermatitis, and reduced kidney immune complexes.

Conclusions Conclusions: Human and murine SLE have evidence of mitochondrial dysfunction, elevated ROS, spontaneous MAVS oligomerization and elevated IFN-I, as well as augmented $\mathrm{T}$ cell homeostatic proliferation. These abnormalities can be largely reversed with MitoQ, with alleviation of disease in murine SLE.

Funding Source(s): NIH (NIAID, NIAMS, NIGMS)

\section{MYOCARDIAL IMPAIRMENT ASSESSED BY CARDIAC MAGNETIC RESONANCE IN NEWLY ONSET LUPUS PATIENTS}

${ }^{1}$ Qiang Guo*, ${ }^{1}$ Qingran Yan, ${ }^{2}$ Meng Jiang, ${ }^{3}$ Huihua Ding, ${ }^{4}$ Wei Zhang, ${ }^{3}$ Nan Shen. ${ }^{1}$ Renji Hospital, School of Medicine, Shanghai Jiao Tong University, China; ${ }^{2}$ jiangmeng0919@163. com; ${ }^{3}$ Department of Rheumatology, Renji Hospital, Shanghai Jiao Tong University School of Medicine; ${ }^{4}$ Renji Hospital, School of Medicine, Shanghai Jiao Tong University

\subsection{6/lupus-2019-Ism.39}

Background Exploring early warning signs of cardiac involvement in new onset lupus patients by cardiac magnetic resonance (CMR).

Methods We enrolled drug-naïve new onset patients with systemic lupus erythematosus (SLE), and enrolled longstanding SLE and healthy subjects as controls. In terms of CMR, we used T1 mapping and extracellular volume (ECV) to assess extracellular changes; and used late gadolinium enhancement (LGE) to assess regional fibrosis. All the patients received cardiac assessment, in terms of cardiac symptoms, NYHA class, electrocardiogram, echocardiography and lab tests. Lupus disease activity were scored by SELENA-SLEDAI.

Results Fifty patients with drug-naïve new onset SLE, 60 patients with longstanding SLE and 50 healthy subjects were included in three centers. All the naïve patients showed no signs of cardiac impairment according to clinic assessment. Native myocardial T1 and ECV, which are extracellular matrix indices, were elevated in the new onset group (1369 $\pm 79 \mathrm{~ms}$ vs. $1092 \pm 57 \mathrm{~ms}$ in the control group for native $\mathrm{T} 1 ; 32$ $\pm 5 \%$ vs. $24 \pm 3 \%$ in the control group for ECV, $\mathrm{p}<0.001$ respectively). No additional T1 values or ECV elevation was detected in the longstanding SLE group. In contrast, LGE was present more in long-standing than naïve SLE patients (for
LGE present, $12 \%$ vs $40 \%, \mathrm{p}=0.004$; and for LGE size, $0.5 \%$ vs $2.1 \%$ of left ventricle, $p=0.002)$. Our CMR findings including T1 mapping, ECV, myocardial fibrosis index-LGE, seemed irrelevant to general lupus disease activity.

Conclusions This study first indicated that drug-naïve new onset SLE was likely to involve silent cardiac impairment, without positive findings from current clinical rheumatic and cardiac indices. The structural and functional changes in the myocardium were related to the SLE stage. Native myocardial T1 values and ECV could serve as early detection markers of myocardial injury before the presence of visual fibrosis and functional decompensation.

Funding Source(s): NNSF of China 81571535, 81771737; NBRP of China 2014CB541906

\section{ADOLESCENTS PERSPECTIVES ON LIVING WITH CHILDHOOD-ONSET SYSTEMIC LUPUS ERYTHEMATOSUS}

Giya Harry*, Jennifer Huggins, Lori Crosby, Avani Modi. Cincinnati Children's Hospital Medical Center

\subsection{6/lupus-2019-Ism.40}

Background Non-adherence to medical recommendations in childhood-onset systematic lupus erythematosus (cSLE) is estimated to be between 40\%-50\%. For patients with cSLE, nonadherence results in increased hospitalizations, preventable disease damage, disease flares, and higher health care costs. Currently there are no published data regarding adolescent patients perspectives on cSLE. The aim of this study was to (1) identify barriers and facilitators of treatment adherence and (2) characterize the influencers of self-management and adherence for adolescents with cSLE.

Methods Ten adolescents diagnosed with cSLE per ACR SLE classification criteria were recruited from a pediatric rheumatology clinic and cSLE patient registry. Participants characteristics are summarized in table 1. Two separate focus groups were conducted and led by trained facilitators to discuss topics around living with cSLE and its management. The sessions were audio-taped, transcribed, and coded for themes by three independent coders.

Results Focus group transcript analyses revealed seven major themes: 1) Barriers/Facilitators of treatment adherence, 2) Symptoms Impacting Daily Life, 3) Lack of Understanding/ Knowledge about cSLE, 4) Impact on personal relationships, 5) Self-care and management, 6) Worry about the Future, and (7) Relationship/communication with healthcare providers. Adherence barriers included the number, taste, timing and side effects of oral medications. Adherence facilitators were desire to avoid being sick/in pain/hospitalized, use of pill boxes, reminder apps, and storage location of pills. Fatigue, pain, and mood significantly impacted daily life for these adolescents. All adolescents described difficulties at school, including absenteeism related to disease flares and hospitalizations, missed school work, bullying, and/or the general lack of awareness and understanding of cSLE among educators and peers. They emphasized the role of healthcare providers in helping increase schools awareness and understanding of cSLE. Future worries were quality of life, transition to college, and job opportunities. All adolescents wanted direct communication with, and between, their providers.

Conclusions While adherence was identified as a significant problem, some adolescents also noted strategies to improve 